\title{
CARDIAC VAGAL PREGANGLIONIC NEURONES: AN UPDATE
}

Alexander V Gourine*, Asif Machhada, Stefan Trapp, K. Michael Spyer

Centre for Cardiovascular and Metabolic Neuroscience, Neuroscience, Physiology and Pharmacology, University College London, London WC1E 6BT, UK

Running title: Vagal control of the heart

Key words: atrioventricular conductance, contractility, excitability, left ventricle, parasympathetic, vagal preganglionic neurones

*Corresponding author: Alexander V. Gourine PhD, Neuroscience, Physiology and Pharmacology, University College London, Gower Street, London WC1E 6BT, United Kingdom. Tel/Fax: +44207679 6480. a.gourine@ucl.ac.uk

Acknowledgements: The research in our laboratory referred to in this report was funded by the British Heart Foundation and The Wellcome Trust. MB PhD funding for A.M. was provided by the Medical Research Council and The Rosetrees Trust. A.V.G. is a Wellcome Trust Senior Research Fellow. 


\section{Abstract}

The autonomic nervous system controls the heart by dynamic recruitment and withdrawal of cardiac parasympathetic and sympathetic activities. These activities are generated by groups of sympathoexcitatory and vagal preganglionic neurones residing in a close proximity to each other within well-defined structures of the brainstem. This short essay provides a general overview and an update on the latest developments in our understanding of the central nervous origins and functional significance of cardiac vagal tone. Significant experimental evidence suggests that distinct groups of cardiac vagal preganglionic neurones with different patterns of activity control nodal tissue (controlling the heart rate and atrioventricular conductance) and the ventricular myocardium (modulating its contractility and excitability). 
Neuronal tracing studies conducted in rats (Izzo et al., 1993; Nosaka et al., 1979; Nosaka et al., 1982; Stuesse, 1982; Cheng \& Powley, 2000; Sampaio et al., 2014), cats (Sugimoto et al., 1979; Ciriello et al., 1980; Geis et al., 1980b; Kalia et al., 1980; Bennett et al., 1981; Geis et al., 1981; Miura et al., 1981; Ciriello et al., 1982; Jones et al., 1995; Ford et al., 1990), dogs (Bennett et al., 1981; Hopkins et al., 1982; Hopkins et al., 1984a; Plecha et al., 1988) and pigs (Hopkins et al., 1984b; Hopkins et al., 1997) identified cardiac vagal preganglionic neurones (cVPNs) primarily residing within the brainstem nucleus ambiguus (NA) and the dorsal vagal motor nucleus (DVMN) (Figure 1). Studies on the ontogenesis of the brainstem vagal system in metamorphosing amphibians undergoing anatomical and physiological changes during the transition from water- to air breathing indicate that the DVMN is the primary vagal nucleus. During metamorphosis, rapidly developing cardiorespiratory interactions initiate ventral migration of a subset of DVMN neurones giving rise to a compact formation of the NA which acquires respiratory modulation of activity from the neighbouring respiratory network (Burggren, 1995; Burggren et al., 1994; Smatresk, 1994). The intermediate zone between DVMN and NA is a likely remnant of the migrating population post nuclear division (Jones, 2001; Taylor et al., 1999). The parasympathetic nervous system develops first in the evolution of vertebrates with the sympathetic division appearing relatively late (cardiac sympathetic innervation is not present in elasmobranch fish) (Jones, 2001; Taylor et al., 1999). Therefore, the DVMN is probably the most (evolutionary) ancient CNS structure which harbours autonomic neurones in vertebrates.

\section{Vagal innervation of the heart}

Vagal efferent fibres are well-known to innervate the nodal tissue and the atria controlling both the heart rate, atrioventricular conductance and the strength of atrial contraction (for a review, see (Coote, 2013). The very existence and the functional role of vagal innervation of the cardiac ventricles has been repeatedly questioned, despite clear anatomical evidence showing rich cholinergic innervation of epicardial and endocardial ventricular surfaces in human (Kent et al., 1974; Pauza et al., 2000), pig (Crick et al., 1999; Ulphani et al., 2010) and rat hearts (Zang et al., 2005; Mastitskaya et al., 2012). Evidence for significant parasympathetic innervation of the cardiac 
ventricles were also obtained in experimental studies conducted in cats and dogs where the release of acetylcholine within the ventricular myocardium was recorded during electrical stimulation of the vagus nerve (Eliakim et al., 1961; Akiyama et al., 1994; Akiyama et al., 2001)

\section{Cardiac vagal preganglionic neurones of the nucleus ambiguus}

cVPNs of the NA have rhythmic, respiratory-related patterns of discharge (Figure 1) with B fibre axons innervating the nodal tissue (McAllen et al., 1976; McAllen et al., 1978a; Ciriello et al., 1982). cVPNs have been visualized following retrograde transport of horseradish peroxidase or viral tracers from the cardiac vagal branches or the fat pads that surround the myocardium and contain the post-ganglionic neurones that directly innervate the heart. The morphology of cVPNs is unspectacular as they resemble other motoneurones. These cells have unindented nuclei and their cytoplasm is rich in organelles and in ultrastructural studies are seen to have many synaptic connections on their dendrites (Izzo et al., 1993). The richest afferent input to the NA comes from the nucleus tractus solitarius (NTS) although it also receives inputs from other areas of the brain. There are also indications in some species that there are cVPNs with C fibre axons in the NA (Jordan et al., 1986).

Electrophysiological studies have shown that these VPNs that have an effect on heart rate are localized in the ventrolateral part of the nucleus although some may be distributed more widely (McAllen et al., 1976; 1978b). These represent a subpopulation of the NA neurones that form the cardiac and pulmonary branches of the vagus. In their electrophysiological description of cVPNs, McAllen \& Spyer (McAllen et al., 1976; 1978b) demonstrated that under anaesthesia NA neurones are either silent or have a very low resting discharge rate. However, their discharge at rest, or when facilitated by application of glutamate, is rhythmic. Two major rhythms dominate: the first is correlated with the arterial pulse and is dependent on inputs from the arterial baroreceptors; the second is related to the central respiratory rhythm (Gilbey et al., 1984). The cardiac rhythm is driven by glutamatergic projections from the NTS and is abolished by glutamate receptor antagonists. Powerful baroreceptor modulation and respiratory-related activity distinguishes cVPNs of the NA from the cardiac projecting neurones of the DVMN which are unaffected by these inputs. 
Activation of the arterial chemoreceptors triggers a powerful and complex effect on the NA neurones. The direct effect of chemoreceptor activation is to excite cVPNs if a brief stimulus is delivered during expiration. However the same stimulus delivered during inspiration is ineffective. This resulted in a considerable controversy in regard of the underlying mechanism for the apparent respiratory gating of the chemoreceptor reflex (Daly, 1997). The answer lies in the second key rhythm displayed by the cVPNs of the NA. Whilst synaptic inputs from the arterial baroreceptors largely control the excitability of these neurones, their rhythmic pattern of respiratory-related discharge reflects powerful excitatory and inhibitory inputs from the neighbouring central respiratory oscillator. cVPNs receive an excitatory input during post-inspiration and a powerful inhibitory input during inspiration (Gilbey et al., 1984). These respiratoryrelated changes in cVPN excitability provide a plausible explanation of an apparent 'gating' of other excitatory and inhibitory inputs, including inputs from the peripheral chemoreceptors.

This respiratory patterning of cVPNs discharge and changing sensitivity to other afferent inputs it imposes is of a major physiological significance. It ensures that cardiac output is tightly linked to the respiratory minute volume (Spyer, 1994). Suppression of the respiratory activity as in the diving reflex removes the inspiratory inhibitory input to the cVPNs inducing cardiac slowing. The accompanying fall in the arterial $\mathrm{PO} 2$ in the induced breath-holding will activate the arterial chemoreceptors which exert their excitatory effect on cVPNs producing further bradycardia. Conversely, high respiratory drive as in exercise leads to an enhanced inhibition of cVPNs and increases heart-rate. Any enhancement of the post-inspiratory state, with meditation and yoga for example, will act to lower the heart rate by enhancing cVPNs discharge (this may contribute to the perceived benefits of these behaviours by stabilizing or lowering the arterial blood pressure).

The main physiological role of the NA neurones projecting to the nodal tissue and controlling the heart rate is now firmly established. There are also indications that cVPNs in the NA may have additional effects on cardiac function. Experimental studies conducted in different species (Blinder et al., 2007; Gatti et al., 1996; Sampaio et al., 2014) demonstrated that stimulation of NA neurones could alter the speed of atrioventricular conduction (as evident from changes in P-R interval), independently of 
heart rate changes. Evidence obtained in cats and dogs have also shown that activation of the NA neurones may have a negative inotropic effect (Blinder et al., 1998; Blinder et al., 2007). These studies, however, have provided no indication of the specific properties of these neurones. They may be analogous to the DVMN neurones that control ventricular inotropy (see below) in that they could as well represent the cVPNs projecting to the heart that were shown to have unmyelinated axons in some earlier studies but were not characterised functionally at that time.

\section{Cardiac vagal preganglionic neurones of the dorsal vagal motor nucleus}

Vagal preganglionic neurones residing in the DVMN have $\mathrm{C}$ fibre axons and appear to be insensitive to the peripheral baro- and chemoreceptor inputs or central respiratory drive (Ciriello et al., 1980; Nosaka et al., 1982; Ford et al., 1990; Jones et al., 1998). Electrophysiological studies conducted in vivo and in vitro demonstrated that the majority of DVMN neurones are tonically active at a low and steady firing rate of 0.5-5 $\mathrm{Hz}$ (Figure 1) (Wang et al., 1995; Ballanyi et al., 1996; McTigue et al., 1997). Pacemaker-like activity of DVMN neurones recorded in vitro remains in the presence of tetrodotoxin and manifests as small $\mathrm{Ca}^{2+}$ spikes (Travagli et al., 1991; Yarom et al., 1985). Their action potentials are characterised by a large afterhyperpolarisation that is mediated by a $\mathrm{Ca}^{2+}$-activated $\mathrm{K}^{+}$current (Pedarzani et al., 2000; Sah et al., 1991) indicating that each action potential has a significant $\mathrm{Ca}^{2+}$ component. This was demonstrated by simultaneous recordings of changes in membrane potential and intracellular $\mathrm{Ca}^{2+}$ or pH in these cells (Trapp et al., 1996; Ballanyi et al., 1998;). Most DVMN neurones also exhibit an A-type $\mathrm{K}^{+}$current that delays action potential generation after hyperpolarisation (Yarom et al., 1985). The DVMN receives a large number of modulatory inputs from vagal afferents via projections from the NTS (Powley, 2000) as well as from hypothalamic nuclei and other forebrain areas. The DVMN is populated by a very heterogeneous group of neurones based on the large number of different (mainly subdiaphragmatic) projection targets and a number of studies have reported subtle differences in their electrical properties depending on the projection targets (reviewed in Travagli et al., 2006). However, no in vitro study has specifically investigated the electrical properties of the DVMN neurones projecting to the heart. 
Although studies using injections of viral traces into the fat pads around the cardiac great vessels demonstrated significant DVMN labelling (Standish et al., 1995), the relative proportion of DVMN neurones projecting to the heart is traditionally believed to be low (Jones, 2001). In addition, the magnitude of heart rate decreases triggered by activation of the DVMN is small when compared to bradycardias elicited by stimulation of the cVPNs residing in the NA (for example see Geis et al., 1980a). However, a recent study which demonstrated that selective optogenetic recruitment of the DVMN activity is highly effective in protecting left ventricular myocytes against lethal ischemia/reperfusion injury (Mastitskaya et al., 2012) highlighted the existence of a functionally significant cardiac innervation by the DVMN neuronal projections. These appear to be responsible for parasympathetic modulation of ventricular inotropy and excitability as discussed in detail below.

In dogs, pigs and humans electrical stimulation of the vagus nerve triggers loadindependent decreases in ventricular contractility (Degeest et al., 1964; Degeest et al., 1965; Lewis et al., 2001). In a human study, Landzberg et al. (1994) demonstrated that intracoronary administration of atropine potentiates dobutamine-induced inotropic responses with a loss of the atropine effect in transplanted (i.e. denervated) hearts. These data suggested that the vagus nerve exerts tonic restraining influence on left ventricular contractility. This important conclusion was supported by the results of a recent study conducted in rats by Machhada et al. (2016) who reported that under systemic $\beta$-adrenoceptor blockade combined with spinal cord (C1) transection (to remove sympathetic influences), intravenous administration of atropine increases left ventricular contractility. This effect of atropine was apparent in animals anaesthetised with urethane, but not in animals anaesthetized with pentobarbital, which is consistent with the known effects of these agents on cardiac vagal tone (O'Leary et al., 2003).

Results of the recent studies involving genetic targeting and silencing of the DVMN in a rat model suggested that a significant component of this restraining vagal influence on left ventricular inotropy is provided by tonically active cVPNs of the DVMN. Specific inhibition of the DVMN increases the strength of the ventricular contraction and this effect is not associated with changes in heart rate (Machhada et al., 2016). Functional neuroanatomical mapping, using glutamate and muscimol microinjections to activate or inhibit neuronal cell bodies in distinct locations along the rostro-caudal extent of the 
left and right DVMN indicated that the cVPNs that have an impact on ventricular contractility reside in the caudal region of the left DVMN (Machhada et al., 2016).

An earlier study in rabbits applied vagus nerve stimulation in combination with an anodal block and demonstrated that the recruitment of unmyelinated efferent vagal fibres decreases the strength of the ventricular contraction by 20\% (Garcia Perez et al., 2001). Quantitatively similar effects can be triggered by activation of the neuronal cell bodies in the left caudal DVMN (Machhada et al. 2016), consistent with the hypothesis that tonic vagal influences on the heart are provided by cVPNs with slowly conducting unmyelinated $\mathrm{C}$ fibre axons.

There is also recent evidence suggesting that the cVPNs of the DVMN contribute to the control of ventricular excitability (Machhada et al., 2015). Earlier studies demonstrated that the parasympathetic influence on ventricular refractoriness and arrhythmia threshold is tonic and could be abolished by bilateral vagotomy (see for example (Schwartz et al., 1977)). It appears that a significant component of this tonic restraining vagal influence on the ventricular arrhythmic potential originates from the DVMN. In an anaesthetised rat model, selective acute silencing of the DVMN neurones using a pharmacogenetic approach was found to be associated with shortening of the ventricular effective refractory period (VERP), lowering of the threshold for triggered ventricular tachycardia $\left(\mathrm{V}_{\mathrm{T}}\right)$, and QT interval prolongation (Machhada et al., 2015).

Changes in the electrophysiological properties of the ventricles recorded in conditions of acute DVMN inhibition were remarkably similar to the ventricular electrophysiological phenotype observed in mice with global genetic deletion of the inhibitory G-protein $\mathrm{Ga}_{\mathrm{i} 2}$ (which mediates the downstream signalling cascade triggered by activation of the muscarinic receptors) (Zuberi et al., 2010). However, the data reported by Machhada et al. (2015) also suggests that the DVMN neurones controlling ventricular excitability may recruit cardiac postganglionic neurones which use nitric oxide as a signalling molecule. It was found that inhibition of the DVMN in conditions of combined systemic $\beta$-adrenoceptor and muscarinic receptor blockade was still effective in reducing both the VERP and the $\mathrm{V}_{\mathrm{T}}$ during programmed electrical stimulations of both the left and the right ventricles (Machhada et al., 2015). The evidence of nitric oxide involvement in mediating antiarrhythmic effects of the vagus nerve stimulation was previously reported (Brack et al., 2009; Brack et al., 2011). 
DVMN activity is also critically important for the recruitment of innate mechanisms of inter-organ protection underlying the phenomenon of remote ischaemic conditioning in which episode(s) of ischaemia and reperfusion in tissues remote from the heart reduce myocardial ischaemia/reperfusion injury (Mastitskaya et al., 2012; Gourine \& Gourine, 2014). DVMN neurones may reduce the extent of lethal ischaemia/reperfusion injury either via recruitment of direct cardiac projections and myocardial acetylcholine release (Mastitskaya et al., 2012) or via triggering the production and release into the systemic circulation of certain gut hormones with known cardioprotective properties (Mastitskaya et al., 2016).

\section{Conclusion}

There is strong experimental evidence indicating that distinct populations of cVPNs residing in the NA and the DVMN and displaying different patterns of activity provide differential control of cardiac function. Projections of the NA neurones control the nodal tissue and provide crucial restraining modulation of heart rate. Rhythmic activity of the NA neurones is entrained by the neighbouring respiratory network and underlies the respiratory sinus arrhythmia, therefore, contributing to achieving optimal physiological ventilation/perfusion matching at the level of the lungs. Tonically active neurones of the DVMN are responsible for parasympathetic modulation of ventricular inotropy and excitability and have the potential to protect ventricular cardiomyocytes against ischaemia/reperfusion injury. 


\section{References}

Akiyama, T., Yamazaki, T. 2001. Effects of right and left vagal stimulation on left ventricular acetylcholine levels in the cat. Acta Physiol Scand 172, 11-16.

Akiyama, T., Yamazaki, T., Ninomiya, I. 1994. In vivo detection of endogenous acetylcholine release in cat ventricles. Am J Physiol 266, H854-860.

Ballanyi, K., Doutheil, J., Brockhaus, J. 1996. Membrane potentials and microenvironment of rat dorsal vagal cells in vitro during energy depletion. J Physiol 495, 769-784.

Ballanyi, K., Kulik, A. 1998. Intracellular $\mathrm{Ca}^{2+}$ during metabolic activation of K ${ }_{\text {ATP }}$ channels in spontaneously active dorsal vagal neurons in medullary slices. Eur J Neurosci 10, 2574-2585.

Bennett, J.A., Kidd, C., Latif, A.B., McWilliam, P.N. 1981. A horseradish peroxidase study of vagal motoneurones with axons in cardiac and pulmonary branches of the cat and dog. J Exp Physiol 66, 145-154.

Blinder, K., Dickerson, L., Gray, Lauenstein, J. 1998. Control of negative inotropic vagal preganglionic neurons in the dog: synaptic interactions with substance $P$ afferent terminals in the nucleus ambiguus? Brain Res 810:251-256.

Blinder, K.J., Moore, C.T., Johnson, T.A., Massari, J.V. 2007. Central control of atrioventricular conduction and left ventricular contractility in the cat heart: Synaptic interactions of vagal preganglionic neurons in the nucleus ambiguus with neuropeptide Y-immunoreactive nerve terminals. Auton Neurosci 131, 57-64.

Brack, K.E., Coote, J.H., Ng, G.A. 2011. Vagus nerve stimulation protects against ventricular fibrillation independent of muscarinic receptor activation. Cardiovasc Res 91, 437-446.

Brack, K.E., Patel, V.H., Mantravardi, R., Coote, J.H., Ng, G.A. 2009. Direct evidence of nitric oxide release from neuronal nitric oxide synthase activation in the left ventricle as a result of cervical vagus nerve stimulation. J Physiol 587, 3045-3054.

Burggren, W.W. 1995. Central Cardiovascular Function in Amphibians: Qualitative Influences of Phylogeny, Ontogeny, and Season. In: Heisler, N., (Ed.), Mechanisms of Systemic Regulation, Vol. 21. Springer Berlin Heidelberg. pp. 175-197.

Burggren, W.W., Infantino, R.L. 1994. The Respiratory Transition from Water to AirBreathing during Amphibian Metamorphosis. Am Zool 34, 238-246.

Cheng Z, Powley TL. 2000. Nucleus ambiguus projections to cardiac ganglia of rat atria: An anterograde tracing study. J Comp Neurol 424, 588-606

Ciriello, J., Calaresu, F.R. 1980. Distribution of vagal cardioinhibitory neurons in the medulla of the cat. Am J Physiol 238, R57-64.

Ciriello, J., Calaresu, F.R. 1982. Medullary origin of vagal preganglionic axons to the heart of the cat. J Auton Nerv Syst 5, 9-22.

Coote, J.H. 2013. Myths and realities of the cardiac vagus. J Physiol 591, 4073-4085.

Crick, S.J., Anderson, R.H., Ho, S.Y., Sheppard, M.N. 1999. Localisation and quantitation of autonomic innervation in the porcine heart II: endocardium, myocardium and epicardium. J Anat 195, 359-373. 
Daly M.DeB. 1997. Peripheral arterial chemoreception and respiratory-cardiovascular integration. Monograph for the Physiological Society. Oxford University Press, Oxford.

Degeest, H., Levy, M.N., Zieske, H. 1964. Negative inotropic effect of the vagus nerves upon the canine ventricle. Science 144, 1223-1225.

Degeest, H., Levy, M.N., Zieske, H., Lipman, R.I. 1965. Depression of Ventricular Contractility by Stimulation of the Vagus Nerves. Circ Res 17, 222-235.

Eliakim, M., Bellet, S., Tawil, E., Muller, O. 1961. Effect of vagal stimulation and acetylcholine on the ventricle. Studies in dogs with complete atrioventricular block. Circ Res 9, 1372-1379.

Ford, T.W., Bennett, J.A., Kidd, C., McWilliam, P.N. 1990. Neurones in the dorsal motor vagal nucleus of the cat with non-myelinated axons projecting to the heart and lungs. Exp Physiol 75, 459-473.

Garcia Perez, M., Jordan, D. 2001. Effect of stimulating non-myelinated vagal axons on atrio-ventricular conduction and left ventricular function in anaesthetized rabbits. Auton Neurosci 86, 183-191.

Gatti, P., Johnson, T., Massari, V. 1996. Can neurons in the nucleus ambiguus selectively regulate cardiac rate and atrio-ventricular conduction? J Auton Nerv Syst 57:123-127.

Geis, G.S., Kozelka, J.W., Wurster, R.D. 1981. Organization and reflex control of vagal cardiomotor neurons. J Auton Nerv Syst 3, 437-450.

Geis, G.S., Wurster, R.D. 1980a. Cardiac responses during stimulation of the dorsal motor nucleus and nucleus ambiguus in the cat. Circ Res 46, 606-611.

Geis, G.S., Wurster, R.D. 1980b. Horseradish peroxidase localization of cardiac vagal preganglionic somata. Brain Res 182, 19-30.

Gilbey, M.P., Jordan, D., Richter, D.W., Spyer, K.M. 1984. Synaptic mechanisms involved in the inspiratory modulation of vagal cardio-inhibitory neurones in the cat. J Physiol 356, 65-78.

Gourine, A., Gourine, A.V. 2014. Neural mechanisms of cardioprotection. Physiology (Bethesda) 29, 133-140.

Hopkins, D.A., Armour, J.A. 1982. Medullary cells of origin of physiologically identified cardiac nerves in the dog. Brain Res Bull 8, 359-365.

Hopkins, D.A., Armour, J.A. 1984a. Localization of sympathetic postganglionic and parasympathetic preganglionic neurons which innervate different regions of the dog heart. J Comp Neurol 229, 186-198.

Hopkins, D.A., Gootman, P.M., Gootman, N., Armour, J.A. 1997. Anatomy of medullary and peripheral autonomic neurons innervating the neonatal porcine heart. J Auton Nerv Syst 64, 74-84.

Hopkins, D.A., Gootman, P.M., Gootman, N., Russo, S.M., Zeballos, M.E. 1984b. Brainstem cells of origin of the cervical vagus and cardiopulmonary nerves in the neonatal pig (Sus scrofa). Brain Res 306, 63-72.

Izzo, P.N., Deuchars, J., Spyer, K.M. 1993. Localization of cardiac vagal preganglionic motoneurones in the rat: immunocytochemical evidence of synaptic inputs containing 5-hydroxytryptamine. J Comp Neurol 327, 572-583.

Jones, J.F. 2001. Vagal control of the rat heart. Exp Physiol 86, 797-801. 
Jones, J.F., Wang, Y., Jordan, D. 1995. Heart rate responses to selective stimulation of cardiac vagal $C$ fibres in anaesthetized cats, rats and rabbits. J Physiol 489, 203-214.

Jones, J.F., Wang, Y., Jordan, D. 1998. Activity of C fibre cardiac vagal efferents in anaesthetized cats and rats. J Physiol 507, 869-880.

Jordan, D., Spyer, K.M. 1986. Brainstem integration of cardiovascular and pulmonary afferent activity. Prog Brain Res 67, 295-314.

Kalia, M., Mesulam, M.M. 1980. Brain stem projections of sensory and motor components of the vagus complex in the cat: II. Laryngeal, tracheobronchial, pulmonary, cardiac, and gastrointestinal branches. J Comp Neurol 193, 467-508.

Kent, K.M., Epstein, S.E., Cooper, T., Jacobowitz, D.M. 1974. Cholinergic innervation of the canine and human ventricular conducting system. Anatomic and electrophysiologic correlations. Circulation 50, 948-955.

Landzberg, J.S., Parker, J.D., Gauthier, D.F., Colucci, W.S. 1994. Effects of intracoronary acetylcholine and atropine on basal and dobutamine-stimulated left ventricular contractility. Circulation 89, 164-168.

Lewis, M.E., Al-Khalidi, A.H., Bonser, R.S., Clutton-Brock, T., Morton, D., Paterson, D., Townend, J.N., Coote, J.H. 2001. Vagus nerve stimulation decreases left ventricular contractility in vivo in the human and pig heart. J Physiol 534, 547-552.

Machhada, A., Ang, R., Ackland, G.L., Ninkina, N., Buchman, V.L., Lythgoe, M.F., Trapp, S., Tinker, A., Marina, N., Gourine, A.V. 2015. Control of ventricular excitability by neurons of the dorsal motor nucleus of the vagus nerve. Heart Rhythm 12, 22852293.

Machhada, A., Marina, N., Stuckey, J., Lythgoe, M.F., Gourine, A.V. 2016. Origins of the vagal drive controlling left ventricular contractility. J Physiol (in Press).

Mastitskaya, S., Basalay, M., Hosford, P.S., Ramage, A.G., Gourine, A., Gourine, A.V. 2016. Identifying the source of a humoral factor of remote (pre)conditioning cardioprotection. PLoS One 11, e0150108.

Mastitskaya, S., Marina, N., Gourine, A., Gilbey, M.P., Spyer, K.M., Teschemacher, A.G., Kasparov, S., Trapp, S., Ackland, G.L., Gourine, A.V. 2012. Cardioprotection evoked by remote ischaemic preconditioning is critically dependent on the activity of vagal pre-ganglionic neurones. Cardiovasc Res 95, 487-494.

McAllen, R.M., Spyer, K.M. 1976. The location of cardiac vagal preganglionic motoneurones in the medulla of the cat. J Physiol 258, 187-204.

McAllen, R.M., Spyer, K.M. 1978a. Two types of vagal preganglionic motoneurones projecting to the heart and lungs. J Physiol 282, 353-364.

McAllen, R.M., Spyer, K.M. 1978b. The baroreceptor input to cardiac vagal motoneurones. J Physiol 282 365-374.

McTigue, D.M., Hermann, G.E., Rogers, R.C. 1997. Effect of pancreatic polypeptide on rat dorsal vagal complex neurons. J Physiol 499, 475-483.

Miura, M., Okada, J. 1981. Cardiac and non-cardiac preganglionic neurones of the thoracic vagus nerve: an HRP study in the cat. Jpn J Physiol 31, 53-66.

Nosaka, S., Yamamoto, T., Yasunaga, K. 1979. Localization of vagal cardioinhibitory preganglionic neurons with rat brain stem. J Comp Neurol 186, 79-92. 
Nosaka, S., Yasunaga, K., Tamai, S. 1982. Vagal cardiac preganglionic neurons: distribution, cell types, and reflex discharges. Am J Physiol 243, R92-98.

O'Leary, D.M., Jones, J.F. 2003. Discharge patterns of preganglionic neurones with axons in a cardiac vagal branch in the rat. Exp Physiol 88, 711-723.

Pauza, D.H., Skripka, V., Pauziene, N., Stropus, R. 2000. Morphology, distribution, and variability of the epicardiac neural ganglionated subplexuses in the human heart. Anat Rec 259, 353-382.

Pedarzani, P., Kulik, A., Muller, M., Ballanyi, K., Stocker, M. 2000. Molecular determinants of $\mathrm{Ca}^{2+}$-dependent $\mathrm{K}^{+}$channel function in rat dorsal vagal neurones. $\mathrm{J}$ Physiol 527 Pt 2, 283-290.

Plecha, D.M., Randall, W.C., Geis, G.S., Wurster, R.D. 1988. Localization of vagal preganglionic somata controlling sinoatrial and atrioventricular nodes. Am J Physiol 255, R703-708.

Powley, T.L. 2000. Vagal circuitry mediating cephalic-phase responses to food. Appetite 34, 184-188.

Sah, P., McLachlan, E.M. 1991. $\mathrm{Ca}^{2+}$-activated $\mathrm{K}^{+}$currents underlying the afterhyperpolarization in guinea pig vagal neurons: a role for $\mathrm{Ca}^{2+}$-activated $\mathrm{Ca}^{2+}$ release. Neuron 7, 257-264.

Sampaio, K.N., Mauad, H., Michael Spyer, K., Ford, T.W. 2014. Chronotropic and dromotropic responses to localized glutamate microinjections in the rat nucleus ambiguus. Brain Res 1542, 93-103.

Schwartz, P.J., Stone, H.L. 1977. Tonic influence of the sympathetic nervous system on myocardial reactive hyperemia and on coronary blood flow distribution in dogs. Circ Res 41, 51-58.

Smatresk, N.J. 1994. Respiratory control in the transition from water to air-breathing in vertebrates. Am Zool 34, 264-279.

Spyer, K.M. 1994. Annual review prize lecture. Central nervous mechanisms contributing to cardiovascular control. J Physiol 474, 1-19.

Standish, A., Enquist, L.W., Escardo, J.A., Schwaber, J.S. 1995. Central neuronal circuit innervating the rat heart defined by transneuronal transport of pseudorabies virus. J Neurosci 15, 1998-2012.

Stuesse, S.L. 1982. Origins of cardiac vagal preganglionic fibers: a retrograde transport study. Brain Res 236, 15-25.

Sugimoto, T., Itoh, K., Mizuno, N., Nomura, S., Konishi, A. 1979. The site of origin of cardiac preganglionic fibers of the vagus nerve: an HRP study in the cat. Neurosci Lett $12,53-58$.

Taylor, E.W., Jordan, D., Coote, J.H. 1999. Central control of the cardiovascular and respiratory systems and their interactions in vertebrates. Physiol Rev 79, 855-916.

Trapp, S., Luckermann, M., Brooks, P.A., Ballanyi, K. 1996. Acidosis of rat dorsal vagal neurons in situ during spontaneous and evoked activity. J Physiol 496, 695-710.

Travagli, R.A., Gillis, R.A., Rossiter, C.D., Vicini, S. 1991. Glutamate and GABAmediated synaptic currents in neurons of the rat dorsal motor nucleus of the vagus. Am J Physiol 260, G531-536. 
Travagli, R.A., Hermann, G.E., Browning, K.N., Rogers, R.C. 2006. Brainstem circuits regulating gastric function. Annu Rev Physiol 68, 279-305.

Ulphani, J.S., Cain, J.H., Inderyas, F., Gordon, D., Gikas, P.V., Shade, G., Mayor, D., Arora, R., Kadish, A.H., Goldberger, J.J. 2010. Quantitative analysis of parasympathetic innervation of the porcine heart. Heart Rhythm 7, 1113-1119.

Wang, Y., Jones, J.F., Ramage, A.G., Jordan, D. 1995. Effects of 5-HT and 5-HT 1 A receptor agonists and antagonists on dorsal vagal preganglionic neurones in anaesthetized rats: an ionophoretic study. $\mathrm{Br}$ J Pharmacol 116, 2291-2297.

Yarom, Y., Sugimori, M., Llinas, R. 1985. Ionic currents and firing patterns of mammalian vagal motoneurons in vitro. Neuroscience 16, 719-737.

Zang, W.J., Chen, L.N., Yu, X.J. 2005. Progress in the study of vagal control of cardiac ventricles. Sheng Li Xue Bao 57, 659-672.

Zuberi, Z., Nobles, M., Sebastian, S., Dyson, A., Lim, S.Y., Breckenridge, R., Birnbaumer, L., Tinker, A. 2010. Absence of the inhibitory G-protein Galphai2 predisposes to ventricular cardiac arrhythmia. Circ Arrhythm Electrophysiol 3, 391-400. 


\section{Figure legends}

\section{Figure 1 | Vagal preganglionic neurones}

A, photomicrograph of the coronal section of the rat brainstem illustrating a representative example of the brainstem distribution of choline acetyltransferase (ChAT)-positive vagal preganglionic neurones of the dorsal motor nucleus of the vagus nerve (DVMN) and the nucleus ambiguus (NA). Neurones of the NA are located in a close proximity to the brainstem respiratory rhythm and pattern generating circuits. XII, hypoglossal motor nucleus (also ChAT-positive); B, schematic drawing of the rat brain in a saggital projection illustrating CNS location and rostro-caudal extent of the DVMN and NA; $\boldsymbol{C}$, schematic illustration drawn from representative electrophysiological recordings of the discharge patterns exhibited by the DVMN (Trapp S, unpublished) and NA (Gilbey et al., 1984) neurones plotted in relation to the activity of the phrenic nerve. 